S. M. Virtanen - M. G. Kenward • M. Erkkola •

S. Kautiainen · C. Kronberg-Kippilä - T. Hakulinen •

S. Ahonen $\cdot$ L. Uusitalo $\cdot$ S. Niinistö $\cdot$ R. Veijola $\cdot$

O. Simell · J. Ilonen $\cdot$ M. Knip

\title{
Age at introduction of new foods and advanced beta cell autoimmunity in young children with HLA-conferred susceptibility to type 1 diabetes
}

Received: 11 December 2005 / Accepted: 14 February 2006 / Published online: 5 April 2006

(C) Springer-Verlag 2006

\begin{abstract}
Aims/hypothesis: Evidence for the role of infant feeding in the development of beta cell autoimmunity is inconsistent. We set out to study the effects of breastfeeding and of age at introduction of supplementary foods on the development of beta cell autoimmunity. Subjects and methods: A prospective birth cohort of 3,565 infants with HLA-DQB1-conferred susceptibility to type 1 diabetes was recruited between 1996 and 2001 from two university hospital areas in Finland. Blood samples were collected at 3 - to 12-month intervals to measure antibodies against islet cells, insulin, glutamate dehydroxylase and islet antigen 2 . The families kept a record on the age at introduction of new foods, and for each visit completed a structured dietary questionnaire. The endpoint was repeated positivity for
\end{abstract}

S. M. Virtanen $(\bowtie) \cdot$ M. Erkkola $\cdot$ C. Kronberg-Kippilä Department of Health Promotion and Chronic Disease

Prevention, National Public Health Institute,

Mannerheimintie 166,

00300 Helsinki, Finland

e-mail: suvi.virtanen@ktl.fi

S. M. Virtanen $\cdot$ M. Erkkola $\cdot$ S. Kautiainen $\cdot$ S. Ahonen

L. Uusitalo

Tampere School of Public Health, University of Tampere,

Tampere, Finland

S. M. Virtanen $\cdot$ S. Kautiainen $\cdot$ S. Ahonen

Tampere University Hospital Research Unit,

Tampere, Finland

M. G. Kenward

Department of Epidemiology and Population Health,

Medical Statistics Unit,

London School of Hygiene and Tropical Medicine,

London, UK

T. Hakulinen

Finnish Cancer Registry,

Helsinki, Finland

S. Niinistö

Department of Public Health, University of Helsinki,

Helsinki, Finland islet cell antibodies together with at least one of the other three antibodies. Results: The overall or exclusive duration of breastfeeding was not associated with the risk of developing the endpoint. An early age at introduction of fruits and berries ( $\leq 4$ months) was related to increased risk of developing positivity for the endpoint (hazard ratio [95\% CI] for earliest tertile 2.02 [1.03-3.95] and for midtertile 1.97 [1.06-3.64] compared with latest tertile $>4$ months). Also, introducing roots between 3 and 3.9 months (midtertile) was related to increased risk of the endpoint (hazard ratio $[95 \% \mathrm{CI}]$ for the earliest tertile 1.04 [0.57-1.90] and for midtertile 1.82 [1.19-2.79] compared with latest tertile). These associations were independent of several putative socio-demographic and perinatal confounding factors. Conclusions/interpretation: Our findings suggest that an early age at introduction of fruits and berries and roots associates independently with beta cell autoimmunity, contradicting earlier findings from smaller birth cohort studies.

R. Veijola

Department of Paediatrics, University of Oulu,

Oulu, Finland

O. Simell

Department of Paediatrics, University of Turku,

Turku, Finland

J. Ilonen

Department of Virology, University of Turku,

Turku, Finland

M. Knip

Department of Paediatrics, Tampere University Hospital,

Tampere, Finland

M. Knip

Hospital for Children and Adolescents, University of Helsinki, Helsinki, Finland

O. Simell $\cdot$ J. Ilonen · M. Knip

The Juvenile Diabetes Research Foundation Center for

Prevention of Type 1 Diabetes in Finland,

Turku, Oulu and Tampere, Finland 
Keywords Aetiology - Beta cell autoimmunity · Infancy · Nutrition - Type 1 diabetes

Abbreviations DIPP: Type 1 Diabetes Prediction and Prevention Project - GADA: $65 \mathrm{kDa}$ isoform of glutamic acid decarboxylase antibodies - IAA: insulin

autoantibodies - IA-2A: tyrosine phosphatase-related islet antigen 2 antibodies $\cdot$ ICA: islet cell antibodies

\section{Introduction}

Evidence for a possible role of early infant feeding patterns in the development of beta cell autoimmunity and clinical type 1 diabetes is inconsistent. In some case-control studies early introduction of cow's milk has been linked to the risk of type 1 diabetes [1], while in two recent prospective birth cohort studies child's age at introduction of cow's milk was not related to the development of at least one of the following type 1 diabetes-associated autoantibodies: insulin autoantibodies (IAA), autoantibodies to the $65 \mathrm{kDa}$ isoform of glutamic acid decarboxylase (GADA), and the tyrosine phosphatase-related islet antigen 2 (IA-2A) [2, 3]. In another study using a nested case-control analysis, the age at introduction of cow's milk was related to the emergence of IA-2A, or to four autoantibodies (islet cell antibodies [ICA], IAA, GADA and IA-2A) [4]. Recently, a putative harmful effect of the early introduction of wheat, rye, oats or barley has been implicated by a German birth cohort study in offspring of parents affected by type 1 diabetes [3], whereas a protective effect of the introduction of these cereals and rice between the age of 4 and 6 months was reported in a Colorado birth cohort of infants at increased genetic risk of type 1 diabetes [2]. It is possible that breastfeeding protects from beta cell autoimmunity or progression to clinical diabetes $[1,5]$.

There are four disease-related autoantibodies that have been shown to predict overt type 1 diabetes: ICA, IAA, GADA and IA-2A [6]. The number of detectable autoantibodies is unequivocally related to the risk of progression to clinical type 1 diabetes in both family studies and general population cohorts [7, 8]. However, previous studies searching for dietary predictors of beta cell autoimmunity have mostly used positivity for one autoantibody only as the endpoint, and the numbers of seroconverters have been small.

We set out to assess whether early introduction of foreign proteins, especially cow's milk and cereals, increases the risk of emergence of autoantibodies associated with type 1 diabetes in a population-based cohort of young children with increased HLA-DQB1-conferred risk of type 1 diabetes. Due to high intercorrelations between age at introduction of different foods in the infant diet, we also evaluated whether the age at introduction of other major foods/food groups is related to the risk of beta cell autoimmunity. Our endpoint was advanced beta cell autoimmunity, i.e. repeated positivity for ICA together with at least one other diabetes-associated major antibody (IAA, GADA or IA-2A).

\section{Subjects and methods}

\section{Subjects}

In the Type 1 Diabetes Prediction and Prevention Project (DIPP), a prospective population-based cohort study [9], newborn infants from the areas of three university hospitals in Finland are screened for HLA-DQB1-conferred susceptibility to type 1 diabetes using samples of cord blood. Infants carrying increased genetic susceptibility (HLADQB $1 * 02 / 0302$ heterozygous and DQB $1 * 0302 /$ subjects; $\mathrm{x}$ stands for homozygosity or a neutral allele) are being monitored for diabetes-associated autoantibodies, growth and viral infections at 3- to 12-month intervals. The families are offered the opportunity to take part in a randomised double-blinded intervention trial with intranasal insulin when their child repeatedly tests positively for at least two autoantibodies. Procedures were approved by the local Ethics Committees. The families gave informed, written consent.

The DIPP Nutrition Study falls within the framework of DIPP. The present series comprises the at-risk children born between 2 September 1996 and 30 June 2001 at Oulu University Hospital and between 20 October 1997 and 30 June 2001 at Tampere University Hospital. In this analysis, data from 3,565 children ( $81 \%$ of the children invited) were available. Of these, $93.5 \%$ were followed up to 6 months, $81.9 \%$ up to 1 year and $68.3 \%$ up to 2 years of age. Of the participants $207(5.8 \%)$ had a first-degree relative with diabetes at the time of the study: 75 a father, 119 a mother and 21 a sibling.

\section{Dietary methods}

Information on infant feeding patterns was collected on each child's diet with 3-, 6-, 12- and 24-month questionnaires as well as with the 'age at introduction of new foods' form. Trained study nurses checked the questionnaires during the respective visits. In the 3-month dietary questionnaire the feeding at the delivery hospital was assessed in detail: whether the child was breast-fed, had received banked breast milk, was exposed to infant formula and if so to which formula, and which of these was/were the main type of feed during the delivery hospital stay. The duration of breastfeeding, the age at introduction of infant formulas and brand names of all formulas the child had received, as well as the age at introduction of other cow's milk products (foods and drinks containing cow's milk or sour milk) were requested in all questionnaires. The formulas were classified as cow's-milk-based, hydrolysed or soy-based. At the age of 3 months all the food items the infant had so far received were carefully recorded. For the first 2 years of the child's life the family was asked to record the age at introduction of all new foods on a continuous form, which was checked at each visit. In the analysis the following food groupings were used: (1) fruits and berries; (2) roots: potato, carrot, turnip, swede; (3) wheat, rye, oats and barley; (4) other cereals: maize, 
Table 1 Characteristics of the participating infants and hazard ratios (HRs) and $95 \%$ CIs of advanced beta cell autoimmunity ${ }^{\mathrm{a}, \mathrm{b}}$ adjusted for genetic risk

\begin{tabular}{|c|c|c|}
\hline Characteristic (number of children with a positive endpoint) & $n(\%)$ & HR $(95 \% \mathrm{CI})$ adjusted for genetic risk \\
\hline Boys (66) & $1,902(53.4)$ & $1.33(0.91-1.95)$ \\
\hline Girls (45) & $1,663(46.6)$ & 1 \\
\hline \multicolumn{3}{|l|}{ HLA-DQB1-conferred risk group } \\
\hline High risk $(\mathrm{DQB} 1 * 02 / * 0302)(35)$ & $684(19.2)$ & $2.04(1.29-3.22)^{\mathrm{c}}$ \\
\hline Moderate risk $\left(\mathrm{DQB} 1 * 0302 / \mathrm{x}^{\mathrm{d}}\right)(76)$ & $2,881(80.8)$ & 1 \\
\hline \multicolumn{3}{|l|}{ Familial diabetes } \\
\hline Yes & $207(5.8)$ & $3.35(2.05-5.50)^{\mathrm{c}}$ \\
\hline No & $3,358(94.2)$ & \\
\hline \multicolumn{3}{|l|}{ Hospital of birth } \\
\hline Oulu $(61)$ & $1,753(49.2)$ & $1.19(0.82-1.73)$ \\
\hline Tampere $(50)$ & $1,812(50.8)$ & 1 \\
\hline \multicolumn{3}{|l|}{ Number of siblings in the family ${ }^{\mathrm{e}}$} \\
\hline None $(38)$ & $1,379(38.7)$ & 1 \\
\hline One (39) & $1,040(29.2)$ & $1.17(0.77-1.78)$ \\
\hline Two or more $(21)$ & $860(24.1)$ & $0.83(0.50-1.37)$ \\
\hline Missing information (13) & $286(8.0)$ & \\
\hline \multicolumn{3}{|l|}{ Maternal basic education $^{\mathrm{e}}$} \\
\hline Less than high-school graduate (40) & $1,582(44.4)$ & 1 \\
\hline High-school graduate (60) & $1,808(50.7)$ & $1.23(0.83-1.84)$ \\
\hline Missing information (11) & $175(4.9)$ & \\
\hline \multicolumn{3}{|l|}{ Maternal age (years) ${ }^{\mathrm{e}}$} \\
\hline$<25(23)$ & $692(19.4)$ & 1 \\
\hline $25-29(32)$ & $1,193(33.5)$ & $0.69(0.41-1.17)$ \\
\hline$\geq 30(56)$ & $1,680(47.1)$ & $0.83(0.52-1.33)$ \\
\hline \multicolumn{3}{|l|}{ Gestational age (weeks) $^{\mathrm{b}}$} \\
\hline 1st quartile: <39 (37) & 869 & 1 \\
\hline 2nd quartile: 39 (21) & 868 & $0.56(0.33-0.95)^{\mathrm{c}}$ \\
\hline 3rd quartile: $40-40.7$ (30) & 886 & $0.77(0.47-1.24)$ \\
\hline 4th quartile: $40.8-(20)$ & 922 & $0.49(0.29-0.85)^{\mathrm{c}}$ \\
\hline Missing information (3) & 20 & \\
\hline \multicolumn{3}{|l|}{ Ponderal index at birth $\left(\mathrm{g} / \mathrm{m}^{3} \times 100\right)$} \\
\hline 1st quartile: $<2.61(26)$ & 888 & 1 \\
\hline 2nd quartile: $2.61-2.77$ (33) & 920 & $1.26(0.75-2.10)$ \\
\hline 3rd quartile: $2.78-2.93$ (25) & 849 & $1.04(0.60-1.80)$ \\
\hline 4th quartile: $2.94-(24)$ & 891 & $0.91(0.52-1.58)$ \\
\hline Missing information (3) & 17 & \\
\hline \multicolumn{3}{|l|}{ Route of delivery } \\
\hline Caesarean section (10) & $454(12.7)$ & $0.69(0.36-1.33)$ \\
\hline Other $(98)$ & $3,094(86.8)$ & 1 \\
\hline Missing information (3) & $17(0.5)$ & \\
\hline \multicolumn{3}{|l|}{ Maternal smoking during pregnancy } \\
\hline Yes $(6)$ & $395(11.1)$ & $0.52(0.23-1.18)$ \\
\hline No $(100)$ & $3,060(85.8)$ & 1 \\
\hline Missing information (5) & $110(3.1)$ & \\
\hline Total $(111)$ & 3,565 & \\
\hline
\end{tabular}

\footnotetext{
${ }^{a}$ A piece-wise exponential survival model was used in the analysis. ICA were used as a primary screening tool. All samples of the children testing positively at least once for ICA were analysed for IAA, GADA and IA-2A. Repeated positivity for ICA and at least one other autoantibody was regarded as advanced beta cell autoimmunity. Type 1 diabetes was included in the endpoint

${ }^{\mathrm{b}}$ For advanced beta cell autoimmunity the trend across the quartiles was significant $(p<0.05)$ for gestational age ${ }^{\mathrm{c}} p<0.05$

${ }^{x} x$ not equal to $* 02, * 0301, * 0602$

${ }^{\mathrm{e}}$ At the time of the birth of the child
} 
rice, millet or buckwheat; (5) cabbage; (6) milk products and foods containing milk; (7) cow's-milk-based infant formulas (excluding hydrolysed and soy formulas); (8) fish; (9) meat; and (10) sausage. In addition wheat, rye, barley and oats were each analysed separately. Exclusive breastfeeding was defined as the period during which the child received, in addition to breast milk, only water, or vitamin or mineral supplements.

\section{Genetic methods}

HLA-DQB1 alleles were analysed as described earlier [10]. In brief, a part of the second exon of the HLA-DQB1 gene was amplified using a primer pair with a biotinylated 3' primer. The biotinylated PCR products were then transferred to streptavidin-coated microtitration plates, denatured and hybridised with sequence-specific probes labelled with lanthanide chelates: europium, terbium or samarium. Two hybridisation mixtures were used, one containing probes hybridising with $\mathrm{DQB} 1 * 0602$ and *0603, DQB $1 * 0603$ and *0604 and a consensus sequence, and the other containing probes specific for the DQB $1 * 02$, *0301 and *0302 alleles. After appropriate incubations and washings, the specific hybridisation products were detected using three-colour time-resolved fluorescence of the lanthanide chelates.

Immunological methods

Of the four type 1 diabetes-associated autoantibodies analysed, ICA were used as the primary screening tool for beta cell autoimmunity. When a child seroconverted to positivity for ICA for the first time, all the child's preceding (starting from birth) and subsequent samples were analysed for IAA, GADA and IA-2A.

ICA were quantified by a standard indirect immunofluorescence method on sections of frozen human pancreas from a blood group $\mathrm{O}$ donor [11]. The end-point dilution titre of positive samples was recorded and the results expressed in JDF units. The detection limit was 2.5 JDF units. All samples initially positive for ICA were retested for confirmation. The disease sensitivity of the ICA assay in our laboratory was $100 \%$ and the specificity $98 \%$ in the most relevant standardisation workshop round [12]. Serum IAA were quantified with a microassay $[13,14]$ and GADA and IA-2A with specific radiobinding assays $[15,16]$. The IAA values representing the specific binding were expressed in relative units based on a standard curve run on each plate using the MultiCalc software program (Perkin-Elmer Life Sciences Wallac, Turku, Finland) and the GADA and IA-2A values were expressed in relative units based on a standard curve constructed from a dilution of positive and negative samples. The limits for IAA, GADA and IA-2A positivities were set at $1.55,5.35$ and 0.429 relative units, respectively, which represents the 99th percentile among more than 370 non-diabetic Finnish children and adolescents. The disease sensitivity and specificity of the IAA, GADA and IA-2A assays were 44 and $98 \%, 82$ and $98 \%$, and 62 and $100 \%$, respectively, in the Centers for Disease Control sponsored Diabetes Autoantibody Standardisation Programme Workshop 2002. Samples with IAA, GADA or IA-2A values between the 97.5th and 99.5th percentiles were reanalysed to confirm the antibody status. Transplacentally transferred autoantibodies [17] were excluded from the analyses. Data on type 1 diabetes-associated autoantibodies until 30 September 2004 were used.

Among the 3,565 children with genetic risk of type 1 diabetes, $237(6.6 \%)$ were positive at least twice for ICA and $101(2.8 \%)$ repeatedly positive for ICA plus at least one other antibody during the median follow-up time of 4 years (range 0.2-8.1 years) since birth. Of the 101 children who were positive for ICA plus at least one other autoantibody, 83 tested positively at least twice for IAA, 66 for GADA and 49 for IA-2A. By 30 September 2004, 42 children $(1.2 \%)$ had progressed to clinical type 1 diabetes at a median age of 3.2 years (range 1.0-6.4 years). Among these, 32 had been repeatedly positive for ICA plus at least one other autoantibody. However, seven of the remaining ten children had or had had one or more autoantibodies in one single sample before or at the time of diagnosis. The three persistently seronegative children had had the last blood sample drawn 2.5, 3.2 and 5.1 years before the diagnosis of diabetes. Therefore we decided to include clinical type 1 diabetes in the autoantibody endpoint. This resulted in 111 children (3.1\% of all children) being positive for the ICA plus at least one other antibody endpoint, i.e. having advanced beta cell autoimmunity, which term will be used throughout the text for the endpoint. None of the children were randomised for the intranasal insulin trial before testing positively for ICA plus at least one other autoantibody. Neither were any of those ten children who were not repeatedly positive for ICA plus at least one other autoantibody, but who developed diabetes, randomised for the intranasal insulin trial.

\section{Socio-demographic and perinatal characteristics}

Information on child's sex, maternal age and education, and the number of siblings was registered by a structured questionnaire completed by the parents after the delivery. Information on duration of gestation, mode of delivery, birth weight and height, and maternal smoking during pregnancy was received from the Medical Birth Registries of the Oulu and Tampere University Hospitals (Table 1).

\section{Statistical methods}

The endpoint (advanced beta cell autoimmunity) is interval-censored and possibly dependent among siblings. To accommodate this structure, a piece-wise exponential survival model was used with constant hazard in the intervals $0-0.99,1-1.99,2-2.99$ and $\geq 3$ years. The results were not sensitive to the particular choice of intervals used. 
Observation intervals beyond positivity did not contribute to the analysis. Random effects for family were introduced to accommodate familial dependence and these were assumed to follow a normal distribution. For some covariates with missing observations the analysis dataset was reduced to such an extent that there were too few siblings to allow sufficiently good estimates of the familyeffect variance to be obtained; on these occasions this component of the model was omitted. In such reduced datasets the impact of familial dependence is anyway minimal. Time-dependent exposures were allowed to influence the risk in a given observational interval only if the exposure could reasonably be expected to affect the subject for at least half of the length of the interval. The models were fitted using maximum-likelihood in SAS PROC NLMIXED, with SEs of estimates derived from the observed information matrix. The proportionality of the hazards was tested by adding interaction terms of the exposure variables with time interval, to the models. The models including interaction terms did not differ largely from the models without them; only for rye and wheat, rye, and oats and barley together was the difference of borderline significance. Logistic regression analysis was used to study background factors associated with age at introduction of selected foods. Predefined categories of the explanatory variables were used: tertiles for all dietary variables and in addition quartiles for the earliest exposures (exclusive breastfeeding and age at introduction of cow's milk). Statistical significance was taken as less than 5\%.

\section{Results}

\section{Baseline characteristics}

Among the baseline characteristics of the study population, only HLA-DQB1-conferred risk, familial diabetes and gestational age were significantly related to advanced beta cell autoimmunity (Table 1 ).

\section{Dietary pattern}

The first supplementary foods given after or in parallel with breast milk were supplementary milk (in $61 \%$ of the children), carrots and potatoes (16\%), and fruits and berries (6\%). The two latter food groups were started at the same time in $9 \%$ and fruits, berries or roots at the same time with supplementary milk in $7 \%$ of the children. Only seven children had first received cereals and ten cereals together with some of the foods mentioned above as the first supplementary food. The total duration of breastfeeding correlated with exclusive breastfeeding $\left(r_{\mathrm{S}}=0.51\right)$ and with age at introduction of most of the foods, e.g. cow's milk products $\left(r_{\mathrm{S}}=0.57\right)$, fruits and berries $\left(r_{\mathrm{S}}=0.44\right)$, roots $\left(r_{\mathrm{S}}=0.50\right)$, the group of wheat, rye, oats and barley $\left(r_{\mathrm{S}}=0.33\right)$, and other cereals $\left(r_{\mathrm{S}}=0.37\right)$. Also the duration of exclusive breastfeeding correlated with the age at introduction of most of the foods, the correlation with age at introduction of cow's milk products being particularly strong $\left(r_{\mathrm{S}}=0.93\right)$. The intercorrelation between age at introduction of cow's milk, fruits and berries, roots, cabbage, wheat, rye, barley and oats, other cereals, fish, meat, sausage and egg varied from 0.17 to 0.49 . The intercorrelations between the age at introduction of wheat, rye, oats and barley products were strong $\left(r_{\mathrm{S}}=0.54-0.80\right)$. Among cereals, oats were introduced first (median age at introduction for oats 5 months, for the others 6 months). The pattern of age at introduction of supplementary milk, fruits and berries, roots, and rye during the first year of life is presented in Fig. 1.

\section{Duration of breastfeeding}

Duration of total and exclusive breastfeeding was not associated with advanced beta cell autoimmunity (Table 2). The results regarding breastfeeding were similar when the information on feeding at the delivery hospital was taken into account (data not shown).

Age at introduction of cow's milk

Several measures of cow's milk exposure were used: any type of cow's milk exposure, exposure to cow's-milk-based infant formulas (hydrolysed and soy-based formulas were excluded), and early exposure in the delivery hospital. The age at any of these first exposures was unrelated to advanced beta cell autoimmunity (Table 2 ).

\section{Age at introduction of fruits and berries and roots}

Early age at introduction of fruits and berries was related to a greater risk of reaching advanced beta cell autoimmunity (Table 2). Age at introduction of roots in the mid-tertile was related to increased risk of advanced beta cell autoimmunity. No evidence of interaction between HLA-DQB1-risk genotypes and age at introduction of fruits and berries or

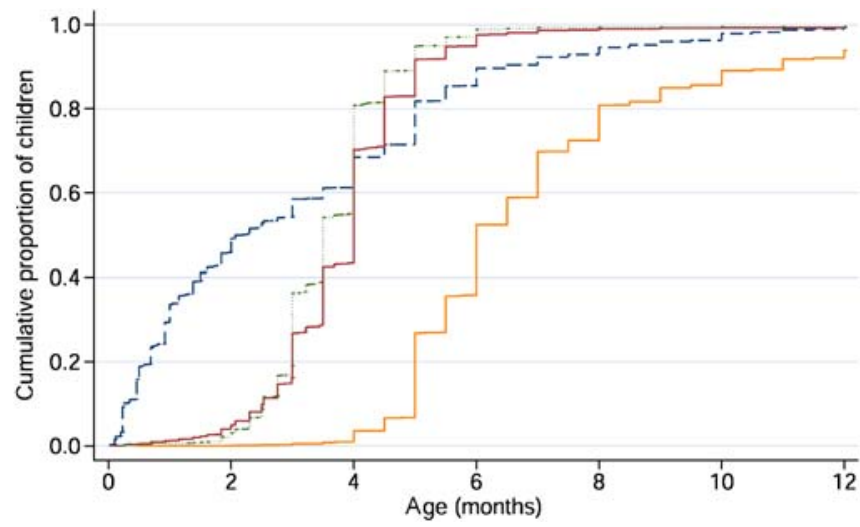

Fig. 1 The cumulative proportion of children $(n=3,565)$ introduced to selected foods by age. Cow's milk includes cow's-milk-based infant formulas. Dashed line, cow's milk; dotted line, roots; unbroken line (purple), fruit and berries; unbroken line (orange), rye 
Table 2 Hazard ratios (HRs) and 95\% CIs adjusted for genetic risk of advanced beta cell autoimmunity related to age at introduction of foods ${ }^{\mathrm{a}, \mathrm{b}}$

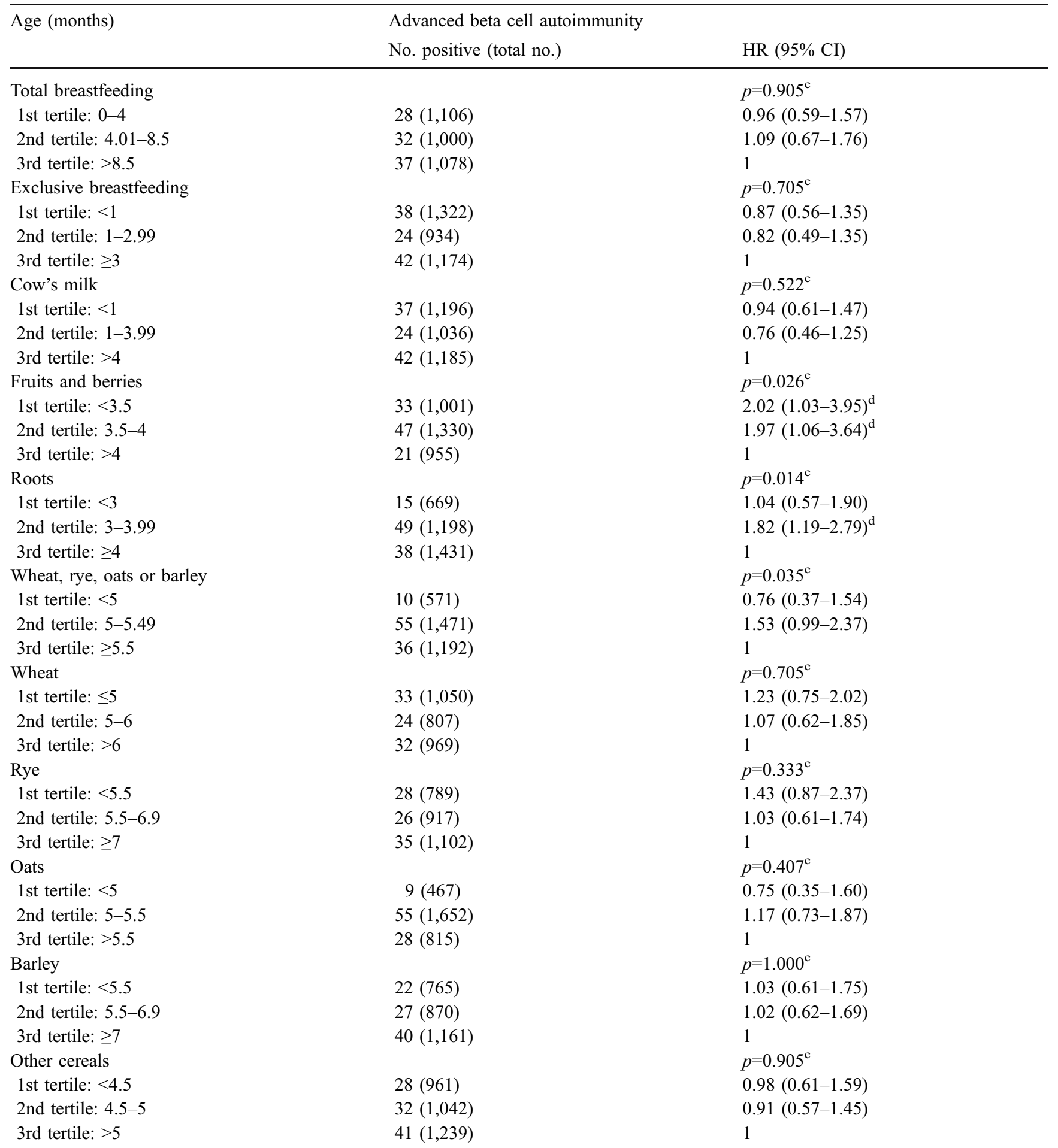

Separate models were used for each dietary variable

${ }^{a}$ A piece-wise exponential survival model was used in the analysis. ICA were used as a primary screening tool. All samples of the children testing positively for ICA were analysed for IAA, GADA and IA-2A. Repeated positivity for ICA and at least one other autoantibody was regarded as advanced beta cell autoimmunity. Type 1 diabetes was included in the endpoint. For breastfeeding variables, age is at the end. The variables were categorised into tertiles. In some cases the same age was reported for many children, which resulted in categories unequal in size. The nearest cut-point was always used

${ }^{\mathrm{b}}$ For advanced beta cell autoimmunity the trend across the tertiles was significant $(p<0.05)$ for combined cereals (wheat, rye, oats, barley), rye alone, fruit and berries, but not for roots

${ }^{c}$ Log likelihood ratio test was used to test whether the model with and without the food variables differed

${ }^{\mathrm{d}} p<0.05$ 
roots was seen in the development of advanced beta cell autoimmunity.

\section{Age at introduction of cereals}

The intake of wheat, rye, oats and barley was not differentiated during the first year of the study (September 1996-August 1997). Using either the combined variable or the specific cereals, no statistically significant associations were observed with advanced beta cell autoimmunity (Table 2).

The introduction of rye products during breastfeeding or during non-breastfeeding did not modify the development of advanced beta cell autoimmunity (data not shown).

\section{Confounding}

Young maternal age, short education and maternal smoking during pregnancy were associated with early introduction (in the earliest tertile) of fruits and berries, roots and rye (data not shown). In addition, boys, children with younger mothers and children living in the Oulu region received fruits and berries and roots earlier than the other children. Low gestational age was related to early introduction of rye, and Caesarean section to early introduction of roots.

When bringing age at introduction of fruits and berries and roots into the same model with socio-demographic and perinatal factors, both food variables retained their significance (Table 3). When both food variables and the socio-demographic and perinatal factors were included in the same model, the association of roots with advanced beta cell autoimmunity remained significant, whereas that of fruits and berries was of borderline significance. The type of the first supplementary food, whether supplementary milk, roots or fruits and berries, was unrelated to advanced beta cell autoimmunity (data not shown).

\section{Discussion}

Our findings from the largest prospective cohort series so far reported suggest that certain patterns of introduction of foods into the infant diet are associated with signs of advanced beta cell autoimmunity in children with increased HLA-conferred genetic susceptibility to type 1 diabetes. In contrast to most previous studies we used as an endpoint repeated positivity for ICA plus at least one other autoantibody, which reflects more advanced and stable beta cell autoimmunity than positivity for only one autoantibody. Young age at the introduction of fruits and berries and of roots was surprisingly related to greater risk of developing the endpoint.

The major virtues of the present study are a well-defined study population, a high participation rate and the use of an endpoint which reflects advanced beta cell autoimmunity. Positivity for a single autoantibody specificity represents in most cases harmless non-progressive beta cell autoimmunity, while the presence of two or more autoantibodies usually reflects a progressive process that only rarely reverts [18]. Positivity for three or four antibodies is associated with a risk of developing clinical type 1 diabetes within a range from 60 to $100 \%$ over the next 5-10 years $[7,8]$. In our study the collection of dietary data before the development of the autoantibody endpoint excluded the possibility of differential bias in the selection of subjects or in the reporting of dietary habits. Unlike previous studies we included in our analyses the age at introduction of all the major foods/food groups and therefore were able to take into account putative confounding effects.

The major limitation of the present study is that only information on the age at introduction of new foods, but not on the amounts of foods consumed could be studied. However, there is increasing evidence that gut-associated lymphoid tissue is involved in the development of type 1 diabetes and as the immune defence mechanisms of the infant gut mature by age [19], the putative dietary regulation of autoimmunity could depend on age. Whether age at introduction of foods is related to later food

Table 3 Hazard ratio (HRs) and 95\% CIs for advanced beta cell autoimmunity associated with the age at introduction of foods ${ }^{\mathrm{a}}$ adjusted for several background factors

\begin{tabular}{lll}
\hline & \multicolumn{2}{l}{${\text { Adjusted } \mathrm{HRs}^{\mathrm{a}}(95 \% \mathrm{CI})}$} \\
\cline { 2 - 3 } & Separate models for fruits/berries and for roots & Model simultaneously including both foods \\
\hline Fruits and berries & $p=0.047^{\mathrm{c}}$ & $p=0.122^{\mathrm{c}}$ \\
1st vs 3rd tertile & $1.90(1.05-3.44)^{\mathrm{b}}$ & $1.99(0.98-4.05)$ \\
2nd vs 3rd tertile & $1.86(1.08-3.19)^{\mathrm{b}}$ & $1.59(0.87-2.93)$ \\
Roots & $p=0.007^{\mathrm{c}}$ & $p=0.011^{\mathrm{c}}$ \\
1st vs 3rd tertile & $0.81(0.41-1.60)$ & $0.52(0.24-1.15)$ \\
2nd vs 3rd tertile & $1.82(1.16-2.85)^{\mathrm{b}}$ & $1.38(0.81-2.34)$ \\
\hline
\end{tabular}

${ }^{\mathrm{a}}$ A piece-wise exponential survival model was used in the analysis. Adjusted for sex, genetic risk, familial diabetes, gestational age, maternal age, educational level and smoking during pregnancy, number of siblings at the time of birth, and for area of birth (Oulu vs Tampere area). ICA were used as a primary screening tool. All samples of the children testing positively for ICA were analysed for IAA, GADA and IA-2A. Repeated positivity for ICA and at least one other autoantibody was regarded as advanced beta cell autoimmunity. Type 1 diabetes was included in the endpoint

${ }^{\mathrm{b}} p<0.05$

${ }^{c}$ Log likelihood ratio test was used to test whether the model with and without the food variables differed 
consumption patterns is not known. The number of children positive for the autoantibody endpoint in our study, although markedly larger than in previous reports in the present field, is still small, causing imprecision in the risk estimates.

The time window for the first exposure is conspicuously narrow in Finland for some of the supplementary foods introduced in infancy, e.g. rye and oats. This reflects the fact that most Finnish parents comply in this respect closely with the national recommendations on infant feeding conveyed by the staff at the Well-Baby Clinics. The narrow age range would make the analyses less sensitive for detecting any risk or protective effects of early exposure to various foods in relation to beta cell autoimmunity.

It can be argued that the dietary exposures measured in our study can act at least partly as proxies of other lifestyle characteristics. In industrialised countries, a low educational attainment, young age and single marital status of the mother, urban environment, having a first baby, smoking and having a baby of male sex are among the factors that associate inversely with total and exclusive duration of breastfeeding [e.g. 20-23]. Less is known about how the choice of weaning foods and the age at introduction of specific foods are related to socio-demographic and perinatal factors. In our cohort of infants, sex, gestational age, maternal age, education and smoking during pregnancy, Caesarean section, number of siblings in the family, and the area of birth were related to the age at introduction of some specific foods in the diet of the child. Other studies suggest that older maternal age is a risk factor for type 1 diabetes [24-28], while the influence of socio-economic status [29], maternal smoking during pregnancy [24, 25], and parental education $[26,29,30]$ remain controversial. The increased risk of developing type 1 diabetes has been linked to improved hygiene and to decreased or changed exposure to infections. Caesarean section [25, 27] and decreased exposure to common infections during infancy [31-33] were associated, although inconsistently [24, 28, $34,35]$, with increased risk of type 1 diabetes, and day-care attendance $[32,34]$ with reduced risk. The adoption of hygiene practices is influenced to some degree by social, lifestyle and environmental factors [36]. Adjustment for several putative confounding socio-demographic, perinatal and dietary factors, however, had minor effects on our results.

On the other hand, it is possible that the observed associations reflect causal relationships. If the observed association of early age at introduction of fruits and berries and roots with advanced beta cell autoimmunity reflects a true relationship, the question arises whether natural or other toxins could be involved in the pathogenesis of type 1 diabetes. Also ingredients added to these baby foods during industrial processing or food preparation at home such as starch or sucrose could play a role. The early introduction of fruits and berries and roots may result in higher energy intake, which could cause beta cell stress and thereby induce beta cell autoimmunity.

Previous cohort studies of children with increased genetic risk have reported associations between age at introduction of cereals and the development of early and potentially reversible beta cell autoimmunity, i.e. positivity for at least one autoantibody $[2,3]$. However, we did not observe a significant association between age at introduction of cereals and the development of ICA plus at least one other autoantibody. Introducing wheat, rye, oats or barley in midtertile (between 5 and 5.49 months) tended to be associated with increased risk of advanced beta cell autoimmunity compared with the latest tertile.

In contrast to previous case-control findings, no relationship was observed between age at introduction of cow's milk and development of advanced beta cell autoimmunity [1]. This is in agreement with previous prospective cohort studies [2-4]. It should be noted, however, that the prospective studies done so far have been underpowered for the detection of such low risk ratios (about 1.5) that have been observed in case-control studies for clinical type 1 diabetes. In the previous nested casecontrol analysis of the DIPP Study [4], early introduction of cow's milk and short duration of exclusive breastfeeding were related to seroconversion to positivity for IA-2A and for all four autoantibodies, a finding which we were not able to confirm in the present analysis with a fivefold higher number of endpoints and using a more accurate dietary data collection, raising the possibility of a chance finding in the previous analysis. Even when using different categorisations applied in other previous studies, no relationship between age at introduction of cow's milk and advanced beta cell autoimmunity was observed (data not shown). Observations from the pilot study of the first nutritional primary prevention study for type 1 diabetes (Trial to Reduce IDDM in the Genetically at Risk, TRIGR) showed that development of ICA or at least one autoantibody can be delayed by giving hydrolysed formula instead of a regular cow's-milk-based one during the first 6-8 months of life in children with at least one family member affected by type 1 diabetes in addition to a riskconferring HLA genotype [37]. It is noteworthy that TRIGR compared hydrolysed formula with a cow's-milkbased one. Such a comparison is not possible in the present observational study. There is some evidence suggesting that the amount of cow's milk consumed could be related to the risk of type 1 diabetes [30, 38-40]. The present analysis did not address quantities but only the age at first exposure.

Although previous analyses [2] have acknowledged the potential relevance of between-sibling dependence, we are the first in this setting to accommodate this dependence formally through an appropriate model; that is, using a frailty term for the family. The variance component associated with this term is not always well estimated with such data, but we have been able to show that its impact on the results is minimal. This is consistent with earlier observations concerning the relevance of betweensibling dependence [2]. In contrast to most previous analyses in this setting we have taken account of the fact that the nutritional covariates are time-dependent. The main consequence of this for our approach is that the introduction of a food at a particular time cannot affect the hazard before the time of introduction. This has 
minimal impact for foods introduced very early, before many seroconversions have occurred, but is more likely to be relevant for foods with a later time of introduction. The use of such a time-dependent analysis does introduce a further complication that requires some approximation: like the outcome itself, the time of introduction of a foodstuff is interval-censored. The following rule was used to accommodate this. A covariate is assumed to affect an individual's hazard in a particular interval if at least half the observational interval overlaps with the covariate exposure. This introduces some inevitable measurement error into the exposure values, but at worst one would expect this to reduce the strength of observed associations, rather than bias these associations in a particular direction. The use of such time-dependent covariates as baseline covariates could conceivably introduce such directional bias through the lack of symmetry in the resulting approximation. Finally, for the principal analyses we have chosen to use predefined categories of the explanatory variables to avoid post hoc or outcome-dependent choices of categorisation.

Our findings from a prospective population-based cohort of individuals with increased genetic risk of type 1 diabetes imply that early age at introduction of fruits and berries and roots is related to the development of advanced beta cell autoimmunity in Finnish children. The significance of this observation remains to be assessed. It is possible that the dietary determinants identified in the present study merely act as a proxy of some other yet unidentified lifestyle or environmental factor/s.

Acknowledgements We express our gratitude to the children and parents who participated, as well as to the DIPP research nurses, doctors, nutritionists and laboratory staff for excellent collaboration over the years. We are also grateful to S. Pohjola, T. Korhonen and J. Hakalax for their skilful technical assistance. The work was supported by the Academy of Finland (grants 63672, 79685,79686, 80846, 201988, 210632), the Finnish Diabetes Association, the Finnish Diabetes Research Foundation, the Finnish Paediatric Research Foundation, the Häme Foundation of the Finnish Culture Fund, the Juho Vainio Foundation, the Yrjö Jahnsson Foundation, Medical Research Funds, Turku, Oulu and Tampere University Hospitals, Juvenile Diabetes Research Foundation (grants 197032, 41998-274, 4-1999-731, 4-200-435), Novo Nordisk Foundation and EU Biomed 2 Program (BMH4-CT98-3314).

\section{References}

1. Virtanen SM, Knip M (2003) Nutritional risk predictors of $\beta$ cell autoimmunity and type 1 diabetes at young age. Am J Clin Nutr 78:1053-1067

2. Norris JM, Barriga K, Klingensmith G et al (2003) Timing of initial cereal exposure in infancy and risk of islet autoimmunity. JAMA 290:1713-1720

3. Ziegler AG, Schmid S, Huber D, Hummel M, Bonifacio E (2003) Early infant feeding and risk of developing type 1 diabetes-associated autoantibodies. JAMA 290:1721-1728

4. Kimpimäki T, Erkkola M, Korhonen S et al (2001) Short-term exclusive breastfeeding predisposes young children with increased genetic risk of type I diabetes to progressive $\beta$ cell autoimmunity. Diabetologia 44:63-69

5. Borch-Johnsen K, Mandrup-Poulsen T, Zachau-Christiansen B et al (1984) Relation between breastfeeding and incidence rates of insulin-dependent diabetes mellitus. Lancet ii:1083-1086
6. Knip M (2002) Can we predict type 1 diabetes in the general population? Diabetes Care 25:623-625

7. Bingley PJ, Bonifacio E, Williams AJK, Genovese S, Bottazzo GF, Gale EAM (1997) Prediction of IDDM in the general populations: strategies based on combinations of autoantibody markers. Diabetes 46:1701-1710

8. LaGasse JM, Brantley MS, Leech NJ et al (2002) Successful prospective prediction of type 1 diabetes in schoolchildren by multiple defined autoantibodies: eight year follow-up of the Washington State Diabetes Prediction Study. Diabetes Care 25:505-511

9. Kupila A, Muona P, Simell T et al (2001) Feasibility of genetic and immunological prediction of type I diabetes in a population-based birth cohort. Diabetologia 44:290-297

10. Ilonen J, Reijonen H, Herva E et al (1996) Rapid HLA-DQB1 genotyping for four alleles in the assessment of risk for IDDM in the Finnish population. Diabetes Care 19:795-800

11. Bottazzo GF, Florin-Christensen A, Doniach D (1974) Islet-cell antibodies in diabetes mellitus with autoimmune polyendocrine deficiencies. Lancet ii:1279-1282

12. Greenbaum CJ, Palmer JP, Nagataki S et al (1992) Improved specificity of ICA assay in the fourth international immunology of diabetes serum exchange workshop. Diabetes 41:1570-1574

13. Ronkainen $\mathrm{M}$, Hämäläinen A-M, Koskela $\mathrm{P}$, Åkerblom HK, Knip M, the Finnish TRIGR Study Group (2001) Pregnancy induces non-immunoglobulin insulin-binding activity in both maternal and cord blood serum. Clin Exp Immunol 124:131136

14. Williams AJK, Bingley PJ, Bonifacio E, Palmer JP, Gale EAM (1997) A novel micro-assay for insulin autoantibodies. J Autoimmun 10:473-478

15. Savola K, Sabbah E, Kulmala P, Vähäsalo P, Ilonen J, Knip M (1998) Autoantibodies associated with type 1 diabetes mellitus persist after diagnosis in children. Diabetologia 41:1293-1297

16. Savola K, Bonifacio E, Sabbah E et al (1998) IA-2 antibodies-a sensitive marker of IDDM with clinical onset in childhood and adolescence. Diabetologia 41:424-429

17. Hämäläinen A-M, Ronkainen MS, Åkerblom HK, Knip M, the Finnish TRIGR Study Group (2000) Postnatal elimination of transplacentally acquired disease-associated antibodies in infants born to families with type 1 diabetes. J Clin Endocrinol Metab 85:4249-4253

18. Knip M (2002) Natural course of preclinical type 1 diabetes. Horm Res 57 [Suppl]:6-11

19. Vaarala O (1999) Gut and induction of immune tolerance in type 1 diabetes. Diabetes Metab Rev 15:353-361

20. North K, Emmett P, Noble S, the ALSPAC Study Team (2000) Types of drinks consumed by infants at 4 and 8 months of age: sociodemographic variations. J Hum Nutr Diet 13:71-82

21. Dubois L, Girard M (2003) Social inequalities in infant feeding during the first year of life. The Longitudinal Study of Child Development in Québec (LSCDQ 1998-2002). Public Health Nutr 6:773-783

22. Yngve A, Sjöström M (2001) Breastfeeding determinants and a suggested framework for action in Europe. Public Health Nutr 4:729-739

23. Pande H, Unwin C, Haheim LL (1997) Factors associated with the duration of breastfeeding: analysis of the primary and secondary responders to a self-completed questionnaire. Acta Paediatr 86:173-177

24. Stene LC, Barriga K, Norris JM et al (2004) Perinatal factors and development of islet autoimmunity in early childhood: the diabetes autoimmunity study in the young. Am J Epidemiol 160:3-10

25. Dahlquist G, Källén B (1992) Maternal-child blood group incompatibility and other perinatal events increase the risk for early-onset type 1 (insulin-dependent) diabetes mellitus. Diabetologia 35:671-675

26. Blom L, Dahlquist G, Nyström L, Sandström L, Wall S (1989) The Swedish childhood diabetes study-social and perinatal determinants for diabetes in childhood. Diabetologia 32:7-13 
27. McKinney PA, Parslow R, Gurney K, Law G, Bodansky HJ, Williams DRR (1997) Antenatal risk factors for childhood diabetes mellitus; a case-control study of medical record data in Yorkshire, UK. Diabetologia 40:933-939

28. Dahlquist GG, Patterson C, Soltesz G (1999) Perinatal risk factors for childhood type 1 diabetes in Europe. The EURODIAB Substudy 2 Study Group. Diabetes Care 22:1698-1702

29. Sipetic S, Vlajinac H, Kocev N, Saji S (2004) The Belgrade childhood diabetes study: prenatal and social associations for type 1 diabetes. Paediatr Perinat Epidemiol 18:33-39

30. Virtanen SM, Hyppönen E, Läärä E et al (1998) Cow's milk consumption, disease associated autoantibodies and type 1 diabetes mellitus: a follow-up study in siblings of diabetic children. Diabet Med 15:730-738

31. Gibbon C, Smith T, Egger P, Betts P, Phillips D (1997) Early infection and subsequent insulin dependent diabetes. Arch Dis Child 77:384-385

32. The EURODIAB Substudy 2 Study Group (2000) Infections and vaccinations as risk factors for childhood type I (insulindependent) diabetes mellitus: a multicentre case-control investigation. Diabetologia 43:47-53

33. Sipetic S, Vlajinac H, Kocev N, Badmanovic S (2003) The Belgrade childhood diabetes study: associations of infections and vaccinations on diabetes in childhood. Ann Epidemiol 13:645-651
34. McKinney PA, Okasha M, Parslow RC et al (2000) Early social mixing and childhood type 1 diabetes mellitus: a case-control study in Yorkshire, UK. Diabet Med 17:236-242

35. Hummel M, Fuchtenbusch M, Schenker M, Ziegler AG (2000) No major association of breast-feeding, vaccinations, and childhood viral diseases with early islet autoimmunity in the German BABYDIAB Study. Diabetes Care 23:969-974

36. Sherriff A, Golding J; Alspac Study Team (2002) Factors associated with different hygiene practices in the homes of 15 month old infants. Arch Dis Child 87:30-35

37. Åkerblom HK, Virtanen SM, Ilonen J et al (2005) Dietary manipulation of $\beta$-cell autoimmunity in infants at increased risk for type 1 diabetes. Diabetologia 48:829-837

38. Verge CF, Howard NJ, Irwig L, Simpson JM, Mackerras D, Silink M (1994) Environmental factors in childhood IDDM. Diabetes Care 17:1381-1389

39. Virtanen SM, Läärä E, Hyppönen E et al (2000) Cow's milk consumption, HLA-DQB1 genotype and IDDM: a nested casecontrol study of siblings of children with diabetes. Diabetes 49:912-917

40. Pundziutè-Lyckå A, Persson L-Å, Cedermark G et al (2004) Diet, growth, and the risk for type 1 diabetes in childhood. Diabetes Care 27:2784-2789 